PII: S0038-1098(97)00013-6

\title{
A STUDY OF LOCALIZATION IN A MODEL SUPERLATTICE WITH 3-DIMENSIONAL DISORDER DISTRIBUTION
}

\author{
P.H. Song, Doochul Kim and D.S. Kim \\ Department of Physics, Seoul National University, Seoul 151-742, Korea
}

(Received 18 October 1996; revised 20 December 1996 by S.G. Louie)

\begin{abstract}
We study a microscopic model for a disordered superlattice system, which simulates the effect of intrinsic alloy fluctuation on localization in alloy superlattices. Numerical investigation of scaling behavior of the localization length shows that excitations along and perpendicular to the superlattice axis undergo a localization transition at a nonzero critical disorder. Therefore, we contend that for localization, the alloy superlattices should be viewed as a 3-dimensional anisotropic material. At the transition, the localization length diverges with critical exponent $\nu=1.2 \pm 0.2$. We expect that the model belongs to the same universality class as the 3 dimensional isotropic Anderson model. (C) 1997 Elsevier Science Ltd. All rights reserved
\end{abstract}

Recently, much attention [1-6] has been paid to the study of the localization transition in anisotropic materials. It seems now that there is general agreement that the critical disorder at which the localization transition takes place and the critical exponent of the localization length are independent of the direction of measurement in spite of the anisotropy.

On the other hand, a careful study by way of a fully 3-dimensional (3D) model is necessary for disordered superlattice (SL) system, where most theoretical studies [7] are based upon 1-dimensional (1D) models. In an ideal SL, i.e. no disorder present, the excitation along the SL axis is decoupled from those in other directions so that it is essentially described by $1 \mathrm{D}$ equations of motion. Almost all electronic and vibrational excitations are known to be localized in 1D space at any nonzero disorder $[8,9]$. Since a certain degree of disorder is always present in real SLs, localization of any excitation is inevitable in the 1D pictures of SL. However, on the experimental side, it has been shown [10] that Bloch transport along the SL axis can take place for relatively small alloy concentration. Moreover, it has been shown [11] that there exists a confined-to-propagating transition of GaAs optical phonons and low energy electronic excitations in $\mathrm{GaAs} / \mathrm{Al}_{x} \mathrm{Ga}_{1-x}$ As $\mathrm{SL}$ as the alloy concentration $x$ is varied. These experimental findings cast doubt on the 1D pictures of SL in the presence of disorder and suggest that for localization disordered SLs should be considered as a 3D anisotropic material.

The impurities in real SL cannot be taken into account fully within 1D models since disorder effects arise not only from randomness along the SL axis but also from the fact that impurities exist in the form of the in-plane (parallel to the SL layers) randomness. In the presence of 3-dimensional (3D) disorder, the three spatial components of an excitation are coupled with one another and the equations of motion do not reduce to 1D ones. A more interesting but rarely investigated case involving the in-plane randomness is the alloy SLs, e.g. $\mathrm{GaAs} / \mathrm{Al}_{x} \mathrm{Ga}_{1-x} \mathrm{As}$. A disorder normally considered in the GaAs/ $/ \mathrm{Al}_{x} \mathrm{Ga}_{1-x} \mathrm{As}$ system is the well-width fluctuation, which is dealt with in 1D picture. The intrinsic alloy disorder cannot be properly treated in this picture and there is no established result for the effect of the 3D alloy scattering on the localization transition.

In this paper, we construct a simple microscopic model for a disordered SL system. In our model, the 3D character of the disorder distribution is fully taken into account, i.e. there exist the in-plane randomness as well as the randomness along the SL axis. Numerical investigation of the scaling behavior of the localization length shows that the excitations along the SL axis 
undergo a localization transition at a nonzero critical disorder, which has not been expected in 1D models. Furthermore, the excitations perpendicular to the SL axis, i.e. in-plane excitations, also become localized at approximately the same value of disorder. At the transition, the localization length diverges with critical exponent $\nu=1.2 \pm 0.2$. We expect that our model belongs to the same universality class as the 3D isotropic Anderson model. Our result suggests that for localization, the excitations of the SL in the presence of in-plane disorder such as the alloy fluctuation should be treated as those in 3D anisotropic materials. This applies to even the "perfect" alloy SL with no well-width fluctuations.

We consider a tight binding Hamiltonian defined on the simple cubic lattice as

$\mathbf{H}=\sum_{\mathbf{r}} \epsilon_{\mathbf{r}}|\mathbf{r}\rangle\left\langle\mathbf{r}\left|+\sum_{\left\langle\mathbf{r}, \mathbf{r}^{\prime}\right\rangle} V\left(|\mathbf{r}\rangle \mathbf{r}^{\prime}|+| \mathbf{r}^{\prime}\right\rangle\langle\mathbf{r}|\right)\right.$,

where $|\mathbf{r}\rangle$ is a Wannier state at the site $\mathbf{r}=\left(n_{x}, n_{y}, n_{z}\right), n_{\alpha}$ being integers and the second sum is over the nearest neighbor pairs. The hopping energies $V$ of a pair of nearest neighbor sites is constant, being set to 1 throughout this work. The on-site energy, $\epsilon_{\mathbf{r}}$, is 0 in alternating layers along the $z$-direction, i.e. for those sites with $n_{z}$ odd, but is a random variable which is uniformly distributed in the interval $(\Delta-W / 2, \Delta+W / 2)$ for sites on layers in between. When $W$ is zero, our model reduces to a SL of the form $A B A B A B \cdots$ along the $z$-direction where the on-site energy of the layer $A$ is zero and that of $B$ is $\Delta$. The SL character of our model comes from the periodic arrangement of the on-site energy. For the ordered system, there exist two SL minibands in our model; the energy is given as

$E=2\left(\cos k_{x}+\cos k_{y}\right)+\frac{\Delta}{2} \pm \sqrt{\frac{\Delta^{2}}{4}+4 \cos ^{2} k_{z}}$,

where the plus (minus) sign is for the upper (lower) branch of the minibands. $k$ represents the index in momentum space. The first term of equation (2) represents the free propagation of electron within SL layers and the last tcrm the energy splitting by the superperiodicity of $2 \times$ (lattice constant). There are two possible interpretations regarding the Wannier state $|\mathbf{r}\rangle$; one is that it represents a unit consisting of group of real atoms, the periodic arrangement of which constructs an SL and the other that it represents a single atomic orbital of an atom at site $\mathbf{r}$. The former is a more commonly used one while in case of the latter, the model (1) may be regarded as a single layer SL system. For $\Delta>6$, the excitation at $E=\Delta$ is situated at the band center of a homogeneous medium constructed solely by the layer $B$ while that by the layer $A$ cannot sustain an excitation at that energy. Therefore by investigating the behavior of the excitation at $E=\Delta$ as $W$ increases from zero, one can study the disorder-induced localization transition.

The quantity of main interest here is the localization length [12]. For a bar-shaped geometry of $L \times L \times N$, the localization length along the $z$-direction, $\lambda_{L}(E, W)$, is calculated from

$\frac{1}{\lambda_{L}(E, W)}=-\lim _{N \rightarrow \infty} \frac{1}{N} \log |G(1, N ; E, W, L)|$

where $G(1, N ; E, W, L)$ designates the Green's function coupling pairs of atoms at opposite ends of the bar of length $N$. We calculate the Green's function recursively by the method of MacKinnon and Kramer [12] for $L \leq 16$. The method can be found in several literatures $[12,13]$, so that only the results of our model calculations are presented below.

Figure 1 shows the $L$-dependence of $\Lambda \equiv \lambda_{L} / L$ for several values of $W$ and for $E=\Delta=9 ; \Delta$ has been chosen to be 9 so that the layer $A$ play a role as periodic barriers. Periodic boundary condition has been imposed in the $x$ - and $y$-directions and only even numbers of $L$ have been considered in our calculation since the data for odd $L$ form distinct sequences from those of even $L$. It can be seen in Fig. 1 that $\log \Lambda$ first decreases as $L$ increases for all values of $W$ presented [14]. This is reasonable since, for smaller $L, 1 \mathrm{D}$ nature of the system would be more dominant and hence one can expect a trend towards localization for all values of $W$. However, $\log \Lambda$ for smaller values of $W$ eventually turns around

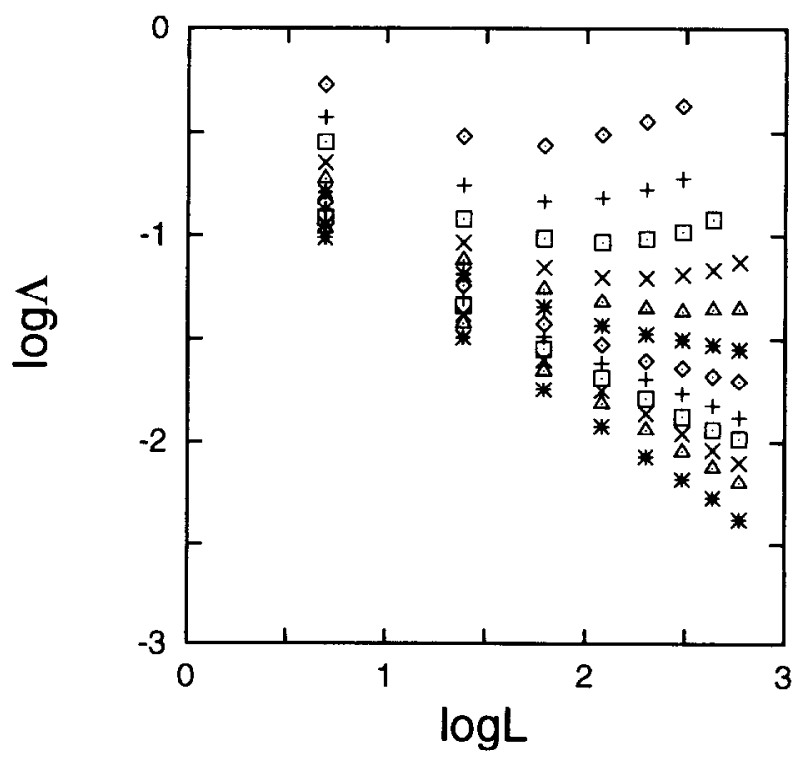

Fig. 1. Log-log plot of the renormalized exponential decay length for excitations along the SL axis as a function of $L$ for various values of $W: W=4(\diamond), 5(+), 6$ $(\bullet), 7(\times), 8(\odot), 9(*), 10(\odot), 11(+), 12(\square), 13(\times)$, $14(\Delta)$ and $16(*)$ from top to bottom. The uncertainty of each data point is less than the symbol size. 
and increases as $L$ increases, which implies that the states are extended in the macroscopic limit. Inverse of the positions of the minimum, $L_{\min }$, in the $\log \Lambda$ vs $L$ curve are shown in Fig. 2 as a function of $W$. It clearly suggests that $L_{\min }$ approaches infinity at a finite value of $W$. This implies the existence of a critical value of $W_{c}$ such that for $W<W_{c}\left(W>W_{c}\right), \log \Lambda$ approaches infinity (minus infinity) as $L \rightarrow \infty$. From the behavior of $L_{\min }$ vs $W$ in Fig. 2, one might say that $W_{c}$, where $1 / L_{\min }=0$, is within the range of $8<W<14$.

In the standard 3D Anderson model [12], similar plots corresponding to Fig. 1 do not show the turn-around behavior present in Fig. 1 and could be analyzed in terms of the scaling form

$\log \Lambda=f\left(\frac{L}{\xi(E, W)}\right)$

with $\xi(E, W) \sim\left|W-W_{c}\right|^{-\nu}$. However, in our case, Fig. 1 suggests that the strict form of equation (4) cannot be applied. In particular, we attribute the turn-around behavior to the effect of the correction-to-scaling terms. We thus assume that the localization length takes the scaling form $[15,16]$

$\lambda_{L}=b F\left[\left(W-W_{c}\right) b^{1 / \nu}, L / b, b^{-\theta} u\right]$

upon the renormalization group transformation where $b$ is the scale change factor, $\theta$ is the leading correction-toscaling exponent and $u$ is the first irrelevant scaling variable. Setting $b=L$ and expanding with respect to $u$, wc obtain

$$
\begin{aligned}
\lambda_{L} / L= & F\left[\left(W-W_{c}\right) L^{1 / \nu}, 1, L^{-\theta} u\right] \\
= & F\left[\left(W-W_{c}\right) L^{1 / \nu}, 1,0\right] \\
& +F^{\prime}\left[\left(W-W_{c}\right) L^{1 / v}, 1,0\right] u / L^{\theta}+\cdots .
\end{aligned}
$$

Keeping only the leading term of the corrections, one

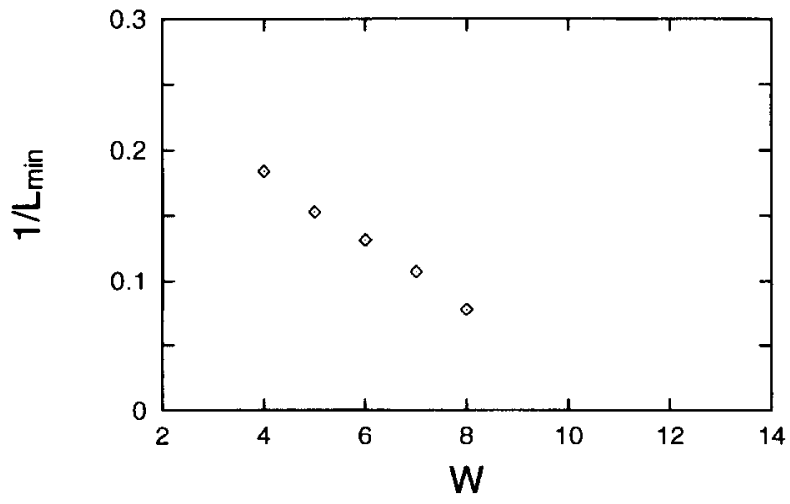

Fig. 2. Plot of $1 / L_{\min }$ vs $W$. The uncertainty of each data point is comparable to the symbol size. sees that equation (4) is modified into the form

$\log \Lambda(W, L)=f\left(\frac{L}{\xi(E, W)}\right)+\frac{c}{L^{\theta}}$,

where $f(x)=\log F\left(x^{1 / \nu}, 1,0\right)$ and $c$ is a constant. At $W=W_{c}$, equation (6) then predicts that

$\log \Lambda\left(W_{c}, L\right)=\log \Lambda_{c}+\frac{c}{L^{\theta}}$,

where $\log \Lambda_{c} \equiv \log \Lambda\left(W_{c}, \infty\right)=f(0)$. We have found that for $L \geq 6$, broad range of data for $9 \leq W \leq 13$ could be fitted reasonably well to the form equation (6). The resulting estimated values of $\log \Lambda_{c}$ and $\theta$ are shown in Table 1, for each tentative values of $W_{c}$. For $W$ near $W_{c}$, equations (6) and (7) implies

$\log \Lambda(W, L)-\log \Lambda\left(W_{c}, L\right)=a\left(W-W_{c}\right) L^{1 / \nu}$,

invoking the usual argument of analyticity of the scaling function in $W$ for finite $L$. Therefore, estimates of $\nu$ can be obtained from the variation of the slopes of $\log \Lambda(W, L)-\log \Lambda\left(W_{c}, L\right)$ with respect to $\left(W-W_{c}\right)$ using data sets $W=W_{c}-1, W_{c}$ and $W_{c}+1$ with $L \geq 6$. The resulting values of $\nu$ are listed in the fourth column of Table 1 . Compared with $\theta$, variation of $\nu$ with the change of estimate of $W$ is smaller, which indicates that the uncertainty in $\nu$ is relatively small regardless of the choice of $W_{c}$.

To determine which set of parameters $\left(W_{c}, \nu\right)$ is close to the true critical values, we examine the scaling plots to see if all the data fall on a same curve with the parameters obtained in the previous procedures. Since the $\nu$ s have been obtained from neighboring data sets of $W s$, i.e. $W=W_{c}-1, W_{c}$ and $W_{c}+1$, it is not guaranteed that other data should scale with the exponent $\nu$ unless $W_{c}$ is chosen correctly. The scaled plots show that the best scaling behavior is obtained for the whole data set of $7 \leq W \leq 16$ when $W_{c}$ is taken as 11 . The result is shown in Fig. 3. The scaling of data is quite good in spite of a somewhat crude approximation of equation (8).

Therefore we have the final estimate of the localization exponent with $W_{c}=11 \pm 1: \nu=1.2 \pm 0.2$. The uncertainty, which has been deduced from Table 1 , represents the typical change in the exponent when $W_{c}$ is varied by unity. We have also performed numerical

Table 1. Values of fitting parameters when tentative value of $W_{c}$ has been chosen as $9,10,11,12$ and 13

\begin{tabular}{llll}
\hline$W_{c}$ & $\log \Lambda_{c}$ & $\theta$ & $\nu$ \\
\hline 9 & -1.63 & 1.24 & 1.35 \\
10 & -1.99 & 0.70 & 1.16 \\
11 & -2.85 & 0.34 & 1.15 \\
12 & -3.67 & 0.24 & 1.37 \\
13 & -9.32 & 0.07 & 1.46 \\
\hline
\end{tabular}




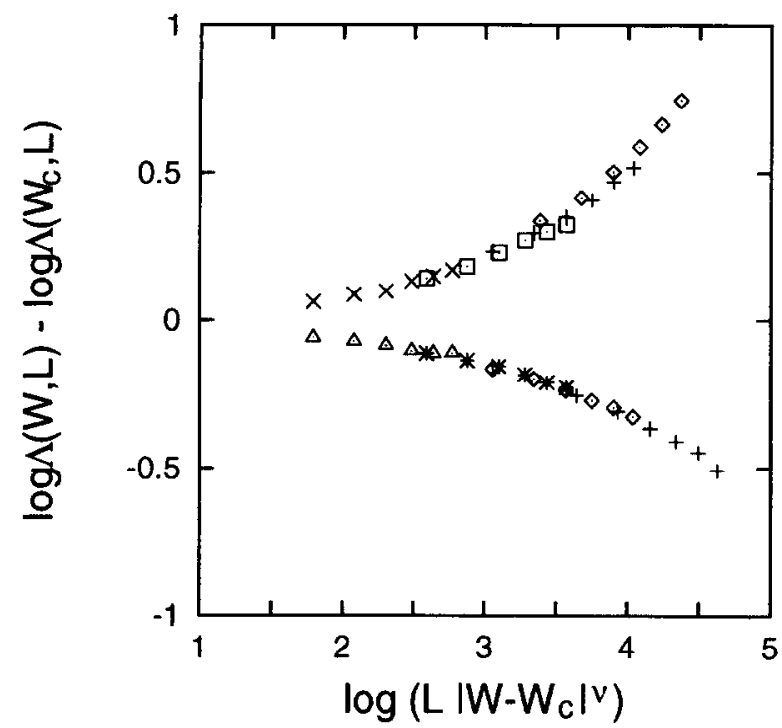

Fig. 3. Scaling behavior of $\log \lambda(W, L)-\log \Lambda\left(W_{c}, L\right)$ with respect to $\log \left(L\left|W-W_{c}\right|^{\nu}\right)$ when $W_{c}$ has been chosen as 11; the data for $W=7(\odot), 8(+), 9(\odot)$ and $10(\times)$ collapse into the upper branch and the data for $W=12(\Delta), 13(*), 14(\diamond)$ and $16(+)$ into the lower branch.

calculation for the localization length of excitations perpendicular to the SL axis. The results are shown in Fig. 4 for the same values of parameters, i.c. $E=\Delta=9$. By the similar procedures as for the excitations along the SL axis, we have found following results: $W_{c, \perp}=10 \pm 1$ with exponent around 1.1-1.5. This is consistent with the

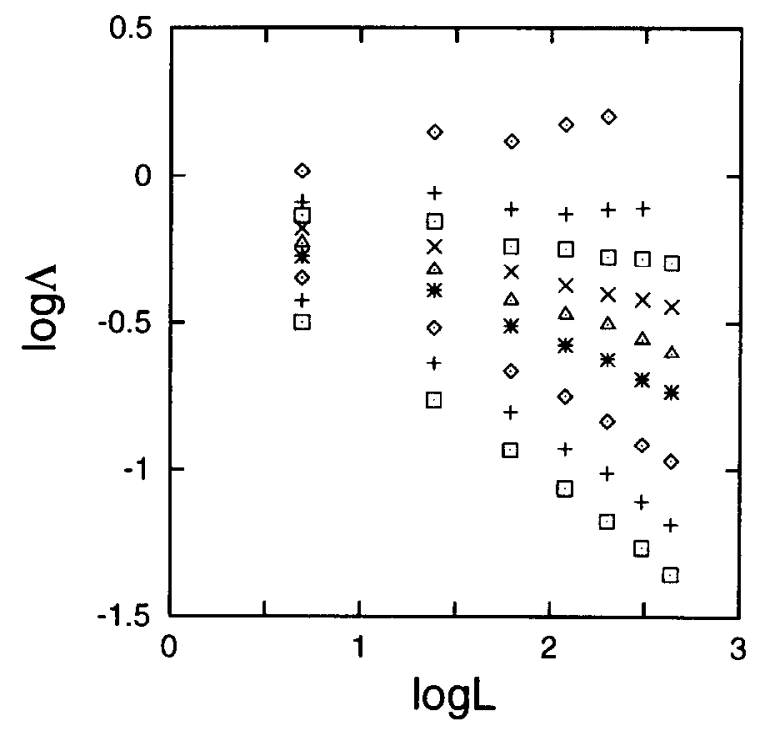

Fig. 4. $\log -\log$ plot of the renormalized exponential decay length for excitations perpendicular to the SL axis as a function of $L$ for various values of $W: W=8$ $(\diamond), 9(+), 9.5(\odot), 10(\times), 10.5(\triangle), 11(*), 12(\diamond), 13$ $(+)$ and $14(\odot)$ from top to bottom. The uncertainty of each data point is less than the symbol size. expectation that the model undergoes the localization transition at the same value of disorder with the same value of $\nu$, regardless of the direction of measurement. These findings are in an accordance with reported results that for anisotropic media, the anisotropy is irrelevant in the renormalization group sense $[1,2,6]$. Estimates of the value of $\nu$ for the 3D isotropic Anderson model varies from $1.2 \pm 0.3$ [12] to $1.5 \pm 0.1$ [9], but more recent value, $1.35 \pm 0.15$ [17] is consistent with our result within the errors. Therefore we expect that our model belongs to the same universality class as the isotropic Anderson model and the anisotropic model of Zambetaki et al. [6].

The 3D feature of localization transition of our SL model suggests that one may consider SL with in-plane disorder as a 3D anisotropic material. Therefore our results provide a microscopic ground for the previous studies $[18,19]$ where a disordered SL system has been treated as an effective anisotropic materials with finite concentration of scattering centers.

An implication of our result for a real SL sample with small degree of impurities is that their contribution to its transport property can be treated simply as a weak perturbation. Since the SL behaves as a 3D material with respect to this kind of disorder, the correction to conductivity induced by the impurities is a well-defined finite quantity even in infinite-size samples at zero temperature. If the transport properties of SL were to follow 1D feature, i.e. localize at arbitrarily small disorder, they would always suffer from the localization effect, which becomes more serious at a larger system size and lower temperature. Therefore, our result justifies the basic assumption that theoretical results for ideal SLs, in which a perfect translational symmetry is assumed, will be valid in real SL samples of sufficiently small disorder or alloy fluctuation.

Our result strongly suggests that the experimentally reported transition [11] of GaAs optical phonons and low energy electronic excitations in $\mathrm{GaAs} / \mathrm{Al}_{x} \mathrm{Ga}_{1-x} \mathrm{As} \mathrm{SL}$ are indeed the localization transitions induced by the intrinsic alloy fluctuation within the layer $\mathrm{Al}_{x} \mathrm{Ga}_{1-x} \mathrm{As}$. Further research including other sources of disorder, e.g. interface roughness, is under progress.

Acknowledgements-We are grateful to Prof. J. Ihm for helpful discussions. This work has been supported by the Korea Science and Engineering Foundation through the Center for Theoretical Physics and by the Ministry of Education through BSRI both at Seoul National University.

\section{REFERENCES}

1. Bhatt, R.N. et al., Phys. Rev., B32, 1985, 569.

2. Qiming, Li et al., Phys. Rev., B40, 1989, 2825. 
3. Rojo, A.G. and Levin, K., Phys. Rev., B48, 1993, 16861 (and references therein).

4. Abrikosov, A.A., Phys. Rev., B50, 1994, 1415.

5. Xue, W. et al., Phys. Rev. Lett., 63, 1989, 2837; Phys. Rev., B42, 1990, 4613; Chu, Q.J. and Zhang, Z.Q., Phys. Rev., B48, 193, 10761.

6. Zambetaki, I. et al., Phys. Rev. Lett., 76, 1996, 3614.

7. Jusserand, B. et al., Phys. Rev., B30, 1984, 6245; Das Sarma, S. et al., Phys. Rev. Lett., 56, 1986, 1280; Tamura, S. and Nori, F., Phys. Rev., B41, 1990, 7941; Chen, X. et al., Phys. Rev., B49, 1994, 14736; Diez, E. et al., Phys. Rev., B50, 1994, 14359; Holthaus, M. et al., Phys. Rev. Lett., 75, $1995,3914$.

8. Abrahams, E. et al., Phys. Rev. Lett., 42, 1979, 673.

9. Kramer, B. and MacKinnon, A., Rep. Prog. Phys., 56, $1993,1469$.

10. Deveaud, B. et al., Phys. Rev. Lett., 58, 1987, 2582.

11. Kim, D.S. et al., Phys. Rev. Lett., 68, 1992, 1002;
Kim, D.S. et al., Phys. Rev., B51, 1995, 5449; Kim, D.S. et al., J. Opt. Soc. Am., B13, 1996, 1210.

12. MacKinnon, A and Kramer, B., Phys. Rev. Lett., 47, 1981, 1546; Z. Phys., B53, 1983, 1.

13. Kalmeyer, V. et al., Phys. Rev., B48, 1993, 11095.

14. On the other hand, for still smaller values of $W$, i.e. less than about $2.38, \log \Lambda$ increases monotonically in $L$. It means that when the data points are interpolated, minimum of $\log \Lambda$ in $L$ is expected to lie in the range of $L<4$ for $W$ smaller than $\sim 2.38$.

15. Privman, V., in Finite Size Scaling and Numerical Simulation of Statistical Systems (Edited by V.Privman), p. 15. World Scientific, Singapore, 1990.

16. Aharony, A. and Hovi, J-P., Phys. Rev. Lett., 72, 1994, 1941.

17. Hoffstetter, E. and Schreiber, M., Europhys. Lett., 21, $1993,933$.

18. Fertig, H.A. and Das Sarma, S., Phys. Rev., B42, $1990,1448$.

19. Szott, W. et al., Phys. Rev., B45, 1992, 3565. 